\title{
Optimization of a Signalized Cell Transmission Model for Network Signal Coordination
}

\author{
C.K. WONG*
}

\author{
Department of Architecture and Civil Engineering, City University of Hong Kong, Tat Chee Avenue, \\ Kowloon Tong, Hong Kong SAR, China \\ wongck@cityu.edu.hk \\ ${ }^{*}$ Corresponding author
}

\begin{abstract}
Keywords: Lane marking design, spatial queue modelling using CTM, and traffic signal coordination.
\end{abstract}

\begin{abstract}
Permitted movements in form of lane markings on single traffic lanes can be defined as decision optimization variables at intersection level and can be connected to form a signalcontrolled network system. Traffic demand patterns are always given in a OD matrix. It is therefore a new design method to find one reliable set of permitted movements across upstream and downstream intersections to serve the OD demand. A lane-based design concept can be extended for the design of a linked signal-controlled network in the sense that the network configuration and signal timings can be optimized simultaneously. Lane-based model conventionally works in a pointqueue system without physical length limits. Incoming vehicles can be queuing along road lanes infinitely. A cell transmission model could be a solution to model spatial queues effectively. With proper signal coordination, vehicles can be released more frequently without exceeding the limited holding capacity of road lanes to avoid overflowing while utilizing green times more efficiently. In this paper, the lane-based design concept and a signalized cell transmission model are integrated to form a new optimization framework. A case study using a pair of T-junctions connecting together will be studied to show the effectiveness of the proposed design approach.
\end{abstract}

\section{Introduction}

Lane-based design method is extending from conventional group-based approach and an enhanced design for signalized intersections [1]. Lane markings or the lane permitted movements binary variables that are optimized with signal timing variables to minimize total delay minimization [2].

It is considered that a network configuration for modelling coordinated signalized intersections is governed by the lane markings. Whether or not a particular OD demand flow can be served to leave a network must be followed by compatible lane marking patterns across upstream and downstream road lanes. Inside a realistic traffic network, road lanes are of different physical lengths and thus having different capacities [3]. Convention point model system does not account the road spatial limits [4]. Queues may be developed infinitely to block back upstream intersections. Practically, overestimating the overall capacity of a signalized system will be resulted pushing the operating signal cycle to its maximum allowable limit. In Hong Kong, it is 120 seconds. An uncontrolled vehicle queues would likely block upstream traffic and cause enormous total delay. A signalized cell transmission model can be formulated as an optimization model to minimize the total delay taking spatial queues into considerations [5]. Shorter cycle times would be designed to discharge vehicles without exceeding the limited holding capacity of road lanes. Optimization results will be the signal settings and lane marking patterns. In this paper, the optimization problem is formulated as a binary-mixed-integer-linear-program and solved by standard branch-and-bound technique to optimize the lane markings to form the network configuration. 


\section{Intersection level}

In this section, the formulation for achieving the optimized lane marking patterns at intersection level is developed. It optimizes the signal timings and lane markings at intersection level.

Permitted movement patterns on lanes. For lane marking patterns, two binary variables, permitted and effective movements are defined. Permitted movements are in form of lane markings painting on ground guiding road users the permitted turn directions on every traffic lane. Safety requirements are given as follows.

$$
1-\Delta_{i, j, k+1} \geq \Delta_{i, m, k}, \quad \forall m=j+1, \ldots, N_{T}-1 ; j=1, \ldots, N_{T}-2 ; k=1, \ldots, L_{i}-1 ; i=1, \ldots, N_{T},
$$

where $\Delta_{i, j, k}=1$ lane marking exists and $=0$ lane marking does not exist. $\Delta_{i, j, k}$ is the permitted movement on lane $k$ for the turn $j$ from arm $i, L_{i}$ is the total number of approach lane on arm $i$, and $N_{T}$ is the total number of traffic arm. Permitted movements are effective movements once demand flows are allocated. To avoid empty usage, a minimum movement is required on a lane to provide at one turning flow as follows.

$$
\sum_{j=1}^{N_{T}-1} \delta_{i, j, k, \pi} \geq 1, \quad \forall k=1, \ldots, L_{i} ; i=1, \ldots, N_{T} ; \pi=1, \ldots, \Pi
$$

where $\delta_{i, j, k, \pi}$ represent effect movements $(=1$, effective movement and $=0$, not effective movement) in a study period $\pi \in \Pi$. Maximum (permitted) movements at stop line is to restrict the possible number of movements on different traffic lanes to eliminate traffic weaving and merging downstream.

$$
E_{\Gamma(i, j)} \geq \sum_{k=1}^{L_{i}} \Delta_{i, j, k}, \quad \forall j=1, \ldots, N_{T}-1 ; i=1, \ldots, N_{T}
$$

where $E_{\Gamma(i, j)}$ is exit lane on arm $\Gamma(i, j)$ from turning movement $\mathrm{j}$ of arm i.

$$
\Delta_{i, j, k} \geq \delta_{i, j, k, \pi}, \quad \forall j=1, \ldots, N_{T}-1 ; k=1, \ldots, L_{i} ; i=1, \ldots, N_{T} ; \pi=1, \ldots, \Pi
$$

Linear constraint set (4) is to ensure only one set of lane marking is established to be the effective lane markings at different time periods.

Signal settings. Cycle time is an important parameter while operating traffic signals. Cycle length $\zeta$ is set between a minimum $C_{\min }$ and a maximum $C_{\max }$ cycle times. Constraints for green start $\theta_{i, j, \pi}$ of turn $\mathrm{j}$ from arm $\mathrm{i}$ in a study period $\pi$ is to ensure that green start time falls inside a signal cycle. Similarly, green durations $\phi_{i, j, \pi}$ for different turns cannot exceed the cycle time and every traffic flow movement must be longer than a minimum green $g_{i, j}$.

$$
\begin{aligned}
& \frac{1}{C_{\min }} \geq \zeta \geq \frac{1}{C_{\max }}, \\
& 1 \geq \theta_{i, j, \pi} \geq 0, \quad \forall j=1, \ldots, N_{T}-1 ; i=1, \ldots, N_{T} ; \pi=1, \ldots, \Pi .
\end{aligned}
$$




$$
1 \geq \phi_{i, j, \pi} \geq g_{i, j} \zeta, \quad \forall j=1, \ldots, N_{T}-1 ; i=1, \ldots, N_{T} ; \pi=1, \ldots, \Pi
$$

Identical signal settings for lanes and turns are given in constraint sets (8) and (9) to match the consistency in displaying the signal settings to road users to avoid ambiguity based on the resulting lane marking patterns.

$$
\begin{aligned}
& M\left(1-\Delta_{i, j, k}\right) \geq \Theta_{i, k, \pi}-\theta_{i, j, \pi} \geq-M\left(1-\Delta_{i, j, k}\right) . \\
& M\left(1-\Delta_{i, j, k}\right) \geq \Phi_{i, k, \pi}-\phi_{i, j, \pi} \geq-M\left(1-\Delta_{i, j, k}\right) .
\end{aligned}
$$

$\Theta_{i, k, \pi}$ and $\Phi_{i, k, \pi}$ are starts and durations of green, respectively on lane $\mathrm{k}$ from arm i and $\mathrm{M}$ is a arbitrarily positive constant number.

Signal displays for conflicting movement pairs must not grant green times at the same period of time to prevent collision. Heydecker (1992) proposed a successor function $\Omega_{i, j, l, m, \pi}$ and Eq. (10) to control relative sequence of incompatible movement groups $(i, j)$ and $(l, m)$.

$$
\Omega_{i, j, l, m, \pi}+\Omega_{l, m, i, j, \pi}=1, \quad \forall((i, j),(l, m)) \in \Psi_{s} ; \pi=1, \ldots, \Pi .
$$

Intergreen times in Eq. (11) are used to separate the right-of-way of conflicting pairs of traffic and the minimum intergreen time is denoted by $\omega_{u, v}$.

$$
\theta_{l, m, \pi}+\Omega_{i, j, l, m, \pi}+M\left(2-\Delta_{i, j, k}-\Delta_{l, m, n}\right) \geq \theta_{i, j, \pi}+\phi_{i, j, \pi}+\omega_{u, v} \zeta, \quad \forall(u, v) \in \Psi, \pi \in \Pi .
$$

Flow related constraint sets. For every design period, the largest demand flow is multiplied by a common flow-multiplier $\mu$. Conservation flow constraint is included in Eq. (12) to equal the total lane flows and input demand flows.

$$
\mu Q_{i, j, \pi}=\sum_{k=1}^{L_{i}} q_{i, j, k, \pi}, \quad \forall j=1, \ldots, N_{T}-1 ; i=1, \ldots, N_{T} ; \pi=1, \ldots, \Pi .
$$

where $Q_{i, j, \pi}$ is the flow demand of turn $\mathrm{j}$ from arm $\mathrm{i}$ in a study period $\pi$, and $q_{i, j, k, \pi}$ is the assigned traffic flow of turn $\mathrm{j}$ from arm $\mathrm{i}$ to be allocated on lane $\mathrm{k}$ in the design period $\pi$. Eq. (13) forces assigned lane flow to zero if lane marking of that turning does not exist. Conversely, if such lane marking exists, then the respective lane flow can be given any non-negative value.

$$
M \delta_{i, j, k, \pi} \geq q_{i, j, k, \pi} \geq 0, \quad \forall k=1, \ldots, L_{i} ; j=1, \ldots, N_{T}-1 ; i=1, \ldots, N_{T} ; \pi=1, \ldots, \Pi .
$$

Constraint set (14) is to force the degree of saturation to be identical for a pair of traffic lanes with a common movement. Their queue lengths must be identical and thus the flow factors must be equal as required.

$$
\begin{gathered}
M\left(2-\delta_{i, j, k, \pi}-\delta_{i, j, k+1, \pi}\right) \geq \frac{1}{s_{i, k}} \sum_{j=1, \ldots, N_{\tau}-1}\left(1+\frac{1.5}{r_{i, j, k}}\right) q_{i, j, k, \pi}-\frac{1}{s_{i, k+1}} \sum_{j=1, \ldots, N_{T}-1}\left(1+\frac{1.5}{r_{i, j, k+1}}\right) q_{i, j, k+1, \pi} . \\
\geq-M\left(2-\delta_{i, j, k, \pi}-\delta_{i, j, k+1, \pi}\right), \quad \forall k=1, \ldots, L_{i}-1 ; i=1, \ldots, N_{T} ; \pi=1, \ldots, \Pi,
\end{gathered}
$$

where $s_{i, k}$ is the lane saturation flow for straight-ahead movement, $r_{i, j, k}$ is turning radius. $p_{i, k}$ in Eq. (15) is the maximum allowable degree of saturation on lane $\mathrm{k}$ in arm i. The optimized green durations for different movements must be long enough to satisfy the minimum requirements.

$$
\Phi_{i, k, \pi}+e \zeta \geq \frac{1}{p_{i, k} s_{i, k}} \sum_{j=1, \ldots, N_{T}-1}\left(1+\frac{1.5}{r_{i, j, k}}\right) q_{i, j, k, \pi}, \forall k=1, \ldots, L_{i} ; i=1, \ldots, N_{T} ; \pi=1, \ldots, \Pi .
$$




\section{Cell Transmission Model}

The following sections are given to develop the proposed cell transmission model to model the spatial queues for each approach and to combine the signalized settings for controlling the traffic flowing in a coordinated signal system from upstream to downstream road lanes.

Signal settings. Minimum red durations in Eq. (16) and minimum green durations Eq. (17) are required to avoid frequent stop and go traffic. Minimum clearance time in Eq. (18-19) is to separate the right-of-way conflicting movements and only one conflicting signal phase can display green to let through traffic.

$$
\begin{aligned}
& -M\left(G_{\bar{i}, \bar{t}}-G_{\bar{i}, \bar{t}+1}-1\right) \geq G_{\bar{i}, \bar{t}+2}+\ldots+G_{\bar{i}, \bar{t}+\bar{r}_{\bar{i}}} \geq M\left(G_{\bar{i}, \bar{t}}-G_{\bar{i}, \bar{t}+1}-1\right), \bar{t}=1, \ldots, \bar{T}-\bar{r}_{\bar{i}} ; \forall \bar{i}, \\
& -M\left(G_{\bar{i}, \bar{t}+1}-G_{\bar{i}, \bar{t}}-1\right) \geq \bar{g}_{\bar{i}}-1-G_{\bar{i}, \bar{t}+2}-\ldots-G_{\bar{i}, \bar{t}+\bar{g}_{\bar{i}}} \geq M\left(G_{\bar{i}, \bar{t}+1}-G_{\bar{i}, \bar{t}}-1\right), \bar{t}=1, \ldots, \bar{T}-\bar{g}_{\bar{i}} ; \forall \bar{i}, \\
& -M\left(G_{\bar{i}, \bar{t}}-G_{\bar{i}, \bar{t}+1}-1\right) \geq G_{\bar{j}, \bar{t}+1}+G_{\bar{j}_{, \bar{t}+2}}+\ldots+G_{\bar{j}, \bar{t}+c_{\bar{i}, \bar{j}}} \geq M\left(G_{\bar{i}, \bar{t}}-G_{\bar{i}, \bar{t}+1}-1\right), \\
& \forall \bar{t}=1, \ldots \bar{T}-c_{\bar{i}, \bar{j}} ; \forall(\bar{i}, \bar{j}) \in \psi . \\
& -M\left(G_{\bar{j}, \bar{t}}-G_{\bar{j}, \bar{t}+1}-1\right) \geq G_{\bar{i}, \bar{t}+1}+G_{\bar{i}, \bar{t}+2}+\ldots+G_{\bar{i}, \bar{t}+c_{\bar{j}, \bar{i}}} \geq M\left(G_{\bar{j}, \bar{t}}-G_{\bar{j}, \bar{t}+1}-1\right), \\
& \forall \bar{t}=1, \ldots \bar{T}-c_{\bar{j}, \bar{i}} ; \forall(\bar{j}, \bar{i}) \in \psi . \\
& G_{\bar{i}, \bar{t}}+G_{\bar{j}, \bar{t}} \leq 1, \forall \bar{t}=1, \ldots \bar{T} ;(\bar{i}, \bar{j}) \in \psi .
\end{aligned}
$$

where $G_{\bar{i}, \bar{t}}=1$ for an effective green and $=0$ for an effective red is the signal setting for signal phase $\bar{i}$ at time step $\bar{t}, \bar{T}$ is the study model period, $\bar{r}_{\bar{i}}$ is the minimum red for signal phase $\bar{i}, \bar{g}_{\bar{i}}$ is the minimum green for signal phase $\bar{i}, c_{\bar{i}}, \bar{j}$ is the minimum clearance time between signal phases $\bar{i}$ and $\bar{j}, \psi$ is a set containing all incompatible signal phases.

Total Demand Input. First CTM cell must be the demand input (source) link storing the total demand. Flow pattern is scheduled to go into a signalized system. The mathematical constraint Eq. (21) is set.

$$
n_{\bar{l}, 1,1}=\bar{\mu} \sum_{\bar{t}=1}^{\bar{T}} d_{\bar{l}, \bar{t}}, \forall \bar{l} \in O,
$$

where $n_{\bar{l}, 1,1}$ denotes the vehicle flow inside first cell of link $\bar{l}$ at first time step, $d_{\bar{l}, \bar{t}}$ is the flow level from link $\bar{l}$ at time step $\bar{t}$, and a common flow multiplier, $\bar{\mu}$, is scaled the demand flows evaluate the system delay.

Input Scheduling. Demand pattern is varying from the OD matrix across different study periods and it can be modeled by varying the exit flow capacities of the first CTM cells for all input links. Constraint set (22) is required in the proposed mathematical framework.

$$
\xi_{\bar{l}, 1, \bar{t}}=\bar{\mu} d_{\bar{l}, \bar{t}}, \bar{t}=1, \ldots, \bar{T} ; \forall \bar{l} \in O .
$$

where $\xi_{\bar{l}, 1, \bar{t}}$ is the exit flow capacity of the first cell on link $\bar{l}$ at time interval $\bar{t}$. The common flow multiplier has scaled the total demand levels and it applies again for consistency. 
Maximum CTM Cell Contents. Last cell $K_{\bar{l}}$ of exit link $\bar{l}(\in D)$ stores all incoming vehicles leaving the system. Holding capacity is infinity. Apart from the last cells, a specific capacity $\varpi$ is used instead to model the physical size of a lane.

$$
\begin{aligned}
& N_{\bar{l}, K_{\bar{l}}, \bar{t}}=\infty, \bar{t}=1, \ldots, \bar{T} ; \forall \bar{l} \in D . \\
& N_{\bar{l}, \bar{k}, \bar{t}}=\varpi, \bar{t}=1, \ldots, \bar{T} ; \bar{k}=2, \ldots, K_{\bar{l}} ; \forall \bar{l} \in O \\
& N_{\bar{l}, \bar{k}, \bar{t}}=\varpi, \bar{t}=1, \ldots, \bar{T} ; \bar{k}=1, \ldots, K_{\bar{l}}-1 ; \forall \bar{l} \in D \\
& N_{\bar{l}, \bar{k}, \bar{t}}=\varpi, \bar{t}=1, \ldots, \bar{T} ; \bar{k}=1, \ldots, K_{\bar{l}} ; \forall \bar{l} \notin\{O, D\}
\end{aligned}
$$

CTM Flow Movements. Traffic turns of different directions move onto downstream cells through the traffic signal settings at intersections. Sent flows $\sigma$ are defined in Eq. (27-29). Network representation uses numerous road lanes. When shared lane markings are designed, a flow proportion $p_{\bar{l}, \bar{l}}$ is necessary to govern the sent flow correctly. $\bar{l}$ is upstream link and $\Gamma(\bar{l})$ gives all downstream links $\bar{l}^{\prime}$. W and $\mathrm{V}$ are the backward wave and free flow speed.

$$
\begin{aligned}
& \sigma_{\bar{l}, \bar{t}} \leq n_{\bar{l}, K_{\bar{l}}, \bar{t}}, \forall \bar{t}=1, \ldots, \bar{T} ; \forall \bar{l} \notin D . \\
& \sigma_{\bar{l}, \bar{t}} \leq \xi_{\bar{l}, K_{\bar{l}}, \bar{t}}, \forall \bar{t}=1, \ldots, \bar{T} ; \forall \bar{l} \notin D . \\
& p_{\bar{l}, \bar{l}} \sigma_{\bar{l}, \bar{t}} \leq \frac{W}{V}\left(N_{\bar{l}^{\prime}, 1, \bar{t}}-n_{\bar{l}^{\prime}, 1, \bar{t}}\right), \forall \bar{t}=1, \ldots, \bar{T} ; \forall \bar{l} \notin D \text { and } \forall \bar{l}^{\prime}=\Gamma(\bar{l}) .
\end{aligned}
$$

Linear constraint sets Eq. (30-36) are standardized to allow smooth transmission of flows.

$$
\begin{aligned}
& n_{\bar{l}, \bar{k}, \bar{t}+1}=n_{\bar{l}, \bar{k}, \bar{t}}+f_{\bar{l}, \bar{k}, \bar{t}}-f_{\bar{l}, \bar{k}+1, \bar{t}}, \bar{t}=1, \ldots, \bar{T}-1 ; k=1, \ldots, K_{\bar{l}}-1 ; \forall \bar{l} \in O . \\
& n_{\bar{l}, K_{\bar{l}}, \bar{t}+1}=n_{\bar{l}, K_{\bar{l}}, \bar{t}}+f_{\bar{l}, K_{\bar{l}}, \bar{t}}-\sigma_{\bar{l}, \bar{t}}, \bar{t}=1, \ldots, \bar{T}-1 ; \forall \bar{l} \notin D . \\
& n_{\bar{l}, K_{\bar{l}}, \bar{t}+1}=n_{\bar{l}, K_{\bar{l}}, \bar{t}}+f_{\bar{l}, K_{\bar{l}}, \bar{t}}, \quad \bar{t}=1, \ldots, \bar{T}-1 ; \forall \bar{l} \in D . \\
& f_{\bar{l}, \bar{k}, \bar{t}} \leq n_{\bar{l}, \bar{k}-1, \bar{t}}, \bar{t}=1, \ldots, \bar{T} ; \bar{k}=2, \ldots, K_{\bar{l}} ; \forall \bar{l} . \\
& f_{\bar{l}, \bar{k}, \bar{t}} \leq \bar{Q}_{\bar{l}, \bar{k}-1, \bar{t}}, \bar{t}=1, \ldots, \bar{T} ; \bar{k}=2, \ldots, K_{\bar{l}} ; \forall \bar{l} . \\
& f_{\bar{l}, \bar{k}, \bar{t}} \leq(W / V)\left(N_{\bar{l}, \bar{k}, \bar{t}}-n_{\bar{l}, \bar{k}, \bar{t}}\right), \bar{t}=1, \ldots, \bar{T} ; \bar{k}=2, \ldots, K_{\bar{l}} ; \forall \bar{l} . \\
& f_{\bar{l}, 1, \bar{t}}=\sum_{\bar{l}^{*}} \lambda_{\bar{l}, \bar{l}^{*}} \sigma_{\bar{l}^{*}, \bar{t}}, \bar{t}=1, \ldots, \bar{T} ; \forall \bar{l} \notin O \text { and } \bar{l}^{*}=\Lambda(\bar{l}) .
\end{aligned}
$$

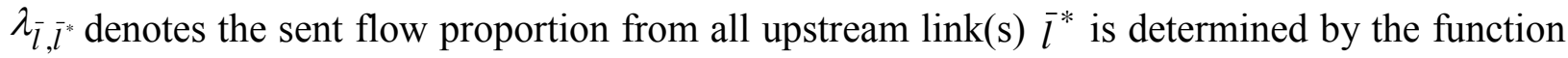
$\Lambda(\bar{l})$ to enter a single downstream link $\bar{l}$. 
CTM Exit Capacity. CTM exit flow denotes the maximum number of vehicle movements. Signalized and ordinary cells are given in constraint sets (37-39). If signal display shows green, full capacity is given for signalized cells. Exit capacity is zero if signal shows red. Z(1) is to identify the signal cell at the end of a link 1 .

$$
\begin{aligned}
& \bar{Q}_{\bar{l}, K_{\bar{l}}, \bar{t}}=G_{\bar{i}, \bar{t}} \bar{s}_{\bar{i}}, \bar{t}=1, \ldots, \bar{T} ; \forall \bar{l} \notin D \text { and } \bar{i}=Z(\bar{l}) . \\
& \bar{Q}_{\bar{l}, \bar{k}, \bar{t}}=\chi_{\bar{l}}, \bar{t}=1, \ldots, \bar{T} ; \bar{k}=2, \ldots, K_{\bar{l}}-1 ; \forall \bar{l} . \\
& \bar{Q}_{\bar{l}, 1, \bar{t}}=\chi_{\bar{l}}, \bar{t}=1, \ldots, \bar{T} ; \forall \bar{l} \notin O .
\end{aligned}
$$

\section{Numerical Example}

Figure 1 shows the configurations of the example network consisting of a pair of T-intersections. L1, L2, L6, and L7 are origin links in the network and L2, L4, L5, and L7 are exit links. Each link comprises 3 approach lanes and shared lane marking patterns are optimized. Demand flow patterns are given by $\mathbf{Q}$ (veh/hr) as follows. L1 and L2 to L3 are $600.0 \mathrm{veh} / \mathrm{hr}$. L2 to L5 is 1,600.0 veh/hr. L6 and L7 to L8 are 1,000 veh/hr. L7 to L4 is $800 \mathrm{veh} / \mathrm{hr}$. Saturation flows $\mathbf{s}$ (veh/hr) take on $6,175.0 \mathrm{veh} / \mathrm{hr}$ for L1 and L6, 5,489 veh/hr for L2 and L7, 5,700 veh/hr for L3 and 5,812 for L8. The lane-based model, $\operatorname{LBM}[(\mathbf{Q}, \Pi) ;(\mathbf{q}, \boldsymbol{\Theta}, \boldsymbol{\Phi}, \boldsymbol{\Omega}, \mathbf{s}, \boldsymbol{\Delta}, \boldsymbol{\delta}, \mu, \zeta)]$ will optimize the flows on different traffic lanes, signal timings, saturation flows, lane marking patterns, common flow multiplier and cycle time for each Tjunction. According to the permitted movements and the saturation flows to form the network configuration in the signalized cell transmission model, SCTM, in Figure 1, the $\operatorname{SCTM}[(\mathbf{q}, \mathbf{s}, \boldsymbol{\Delta}) ;(\mathbf{n}, \mathbf{G}, \bar{\mu})]$ will then optimize the flow transmission patterns including flow values in different CTM cells, the signal settings in each time interval in the present numerical example. Road links of varying saturation flows and signal coordination are modeled, the resulting flow patterns inside CTM cells is more accurate than conventional point-queue framework. There is always an update for the demand flow patterns. The LBM using the updated turning flow patterns as inputs can optimize the lane marking patterns and lane saturation flow levels due to different turning proportions again.

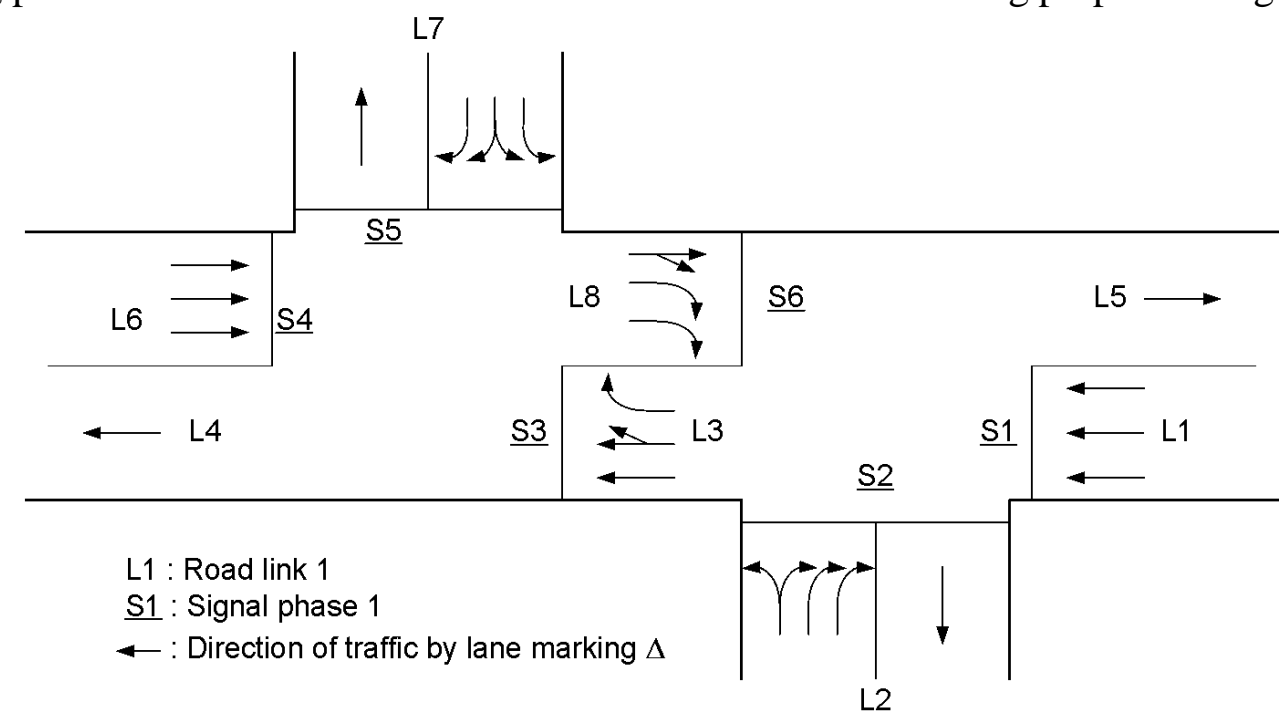

Fig. 1 Optimized Layout of Example Network for Traffic Signal Coordination

The solution process may repeat until convergence Table 1 shows the typical optimization results for the left-hand T-intersection. On each approach lane with lane marking arrows assigned and optimized, assigned lane traffic flows of different turning movements are given in Columns (3-5) to match the input demand flow patterns and total lane flows are the sum of them in Column (6). Column (8) is the lane saturation flows representing the maximum discharging flow rates of traffic 
that would be influenced by the physical lane widths and turning traffic proportion (in Column (7)). Flow factors in Column (9) are the ratios of "Column (6)/Column (8)" by definition and are used to indicate the traffic intensities. Effective green times in Column (10) are then allocated based on these ratios. The ultimate (v/c) demand-to-capacity ratios for all traffic lanes in Column (11) are equalized and reached the prescribed maximum limit of 0.9 in the model optimization.

Table 1 Typical Lane-based CTM modeling result at intersection

\begin{tabular}{|c|c|c|c|c|c|c|c|c|c|c|}
\hline \multirow[t]{2}{*}{$\begin{array}{c}\text { From } \\
\text { Arm } \\
(1)\end{array}$} & $\begin{array}{c}\text { Lane } \\
\text { (2) }\end{array}$ & \multicolumn{3}{|c|}{$\begin{array}{c}\text { To Arm (Assigned lane flow q, } \\
\text { veh/h) }\end{array}$} & \multirow[t]{2}{*}{$\begin{array}{c}\text { Total lane } \\
\text { flow (yeh/h) } \\
\text { (6) }\end{array}$} & \multirow[t]{2}{*}{$\begin{array}{c}\text { Turning } \\
\text { proportion } \\
(7)\end{array}$} & \multirow[t]{2}{*}{$\begin{array}{l}\text { Saturation flow } \\
\text { (veh/h) } \\
(8)\end{array}$} & \multirow[t]{2}{*}{$\begin{array}{c}\text { Flow } \\
\text { factor, } y \\
(9)\end{array}$} & \multirow[t]{2}{*}{$\begin{array}{l}\text { Effective } \\
\text { green (s) } \\
(10)\end{array}$} & \multirow[t]{2}{*}{$\begin{array}{c}\text { Degree of } \\
\text { saturation } \\
(11) \\
\end{array}$} \\
\hline & & 1 & 2 & 4 & & & & & & \\
\hline L7 & 1 & & 572.79 & & 572.79 & $100 \%$ & 1746.7 & 0.3279 & 49.16 & 0.9000 \\
\hline L7 & 2 & & 427.21 & 186.40 & 613.60 & $100 \%$ & 1871.1 & 0.3279 & 49.16 & 0.9000 \\
\hline \multirow[t]{2}{*}{ L7 } & 3 & & & 613.60 & 613.60 & $100 \%$ & 1871.1 & 0.3279 & 49.16 & 0.9000 \\
\hline & & & $(1000.00)$ & $(800.00)$ & & & & & & \\
\hline & & & & & & & & & & \\
\hline L3 & 1 & 12.16 & & 400.0 & 412.16 & $2.95 \%$ & 1957.8 & 0.2105 & 31.56 & 0.9000 \\
\hline L3 & 2 & 393.92 & & & 393.92 & $100 \%$ & 1871.1 & 0.2105 & 31.56 & 0.9000 \\
\hline \multirow[t]{2}{*}{ L3 } & 3 & 393.92 & & & 393.92 & $100 \%$ & 1871.1 & 0.2105 & 31.56 & 0.9000 \\
\hline & & $(800.00)$ & & $(400.00)$ & & & & & & \\
\hline & & & & & & & & & & \\
\hline L6 & 1 & & 318.22 & & 318.22 & $0 \%$ & 1965.0 & 0.1619 & 24.28 & 0.9000 \\
\hline L6 & 2 & & 340.89 & & 340.89 & $0 \%$ & 2105.0 & 0.1619 & 24.28 & 0.9000 \\
\hline \multirow[t]{3}{*}{ L6 } & 3 & & 340.89 & & 340.89 & $0 \%$ & 2105.0 & 0.1619 & 24.28 & 0.9000 \\
\hline & & & $(1000.00)$ & & & & & & & \\
\hline & & & & & & & & & & \\
\hline
\end{tabular}

\section{Conclusions}

The work described in the present paper integrates the lane-based optimization method for isolated signal-controlled junctions and the signalized cell transmission traffic model for linked signal-controlled system. The lane-based optimization results including the permitted movements in the form of lane markings and the lane saturation flows for different individual junctions, part of network configuration, serve as the inputs to signalized cell transmission model for a linked signalcontrolled system. The signalized cell transmission model, taking the spatial capacity of different road links and signal coordination in adjacent signal junctions into consideration, optimizes the coordinated signal settings and traffic flow patterns in the road network. A numerical example of a pair of T-junctions has been used as a demonstration of the optimization procedures and results for practical designs.

\section{Acknowledgments}

This work was supported by a grant from the General Research Fund GRF sponsored by the Research Grants Council of Hong Kong (Project No. 9042062).

\section{References}

[1]. C. K. Wong, B. G. Heydecker, Transport Res B-Meth, 45(4), 667-681 (2011)

[2]. C. K. Wong, Y. Y. Lee, Discrete Dyn Nat Soc, 858731, (2012)

[3]. C. K. Wong, S. C. Wong, J Adv Transport, 36(3), 349-386 (2002)

[4]. S. Lee, S. C. Wong, Y. C. Li, Transport Res C-Emer, 56, 1-17 (2015)

[5]. C. K. Wong, S. C. Wong, H. K. Lo, J Adv Transport, 44(4), 231-244 (2010) 